\title{
ESTUDO DA PREVALÊNCIA DE Ascocotyle (Phagicola) longa EM MUGILÍDEOS CAPTURADOS NA BAÍA DA BABITONGA, SANTA CATARINA, BRASIL
}

\author{
(Ascocotyle (Phagicola) longa prevalence study in mugilides captured from \\ Babitonga Bay, Santa Catarina, Brazil) \\ Juliano Santos Gueretz ${ }^{1}$, Anderson Barbosa de Moura ${ }^{2}$, Maurício Laterça Martins ${ }^{3}$, Antonio Pereira \\ de Souza ${ }^{2}$
}

${ }^{1}$ Instituto Federal Catarinense, Campus Araquari; ${ }^{2}$ Universidade do Estado de Santa Catarina, UDESC CAV Lages; ${ }^{3}$ Universidade Federal de Santa Catarina, Departamento de Aquicultura

Corresponding author: juliano.gueretz@ifc.edu.br

RESUMO: Ascocotyle (Phagicola) longa é o agente etiológico da fagicolose humana, uma zoonose. Os mugilídeos são considerados os segundos hospedeiros intermediários do digenético $A$. (P.) longa, sendo que o primeiro é o caracol Cochliopidae Heleobia australis. Já foram relatadas prevalências de até $100 \%$ de cistos contendo metacercárias de A. (P.) longa, no litoral brasileiro, em Mugil liza, peixes conhecidos como tainhas e amplamente utilizados no consumo humano. $O$ objetivo deste estudo foi verificar a taxa de prevalência de cistos de metacercárias de $A$. (P) longa em mugilídeos, provenientes da Baía da Babitonga, litoral Norte do estado de Santa Catarina. Um total de 119 mugilídeos, sendo 79 espécimes de $M$. curema e $40 \mathrm{M}$. liza, foram adquiridos de pescadores locais, na Baía da Babitonga, no período de novembro de 2015 a outubro de 2017. Amostras de tecido muscular e do fígado de cada peixe foram coletadas para análise parasitológica. As amostras foram fragmentadas em liquidificador, o conteúdo diluído em água foi tamizado e o sedimento analisado em microscópio estereoscópico para pesquisa de cistos de metacercárias de $A$. (P.) longa. Os resultados indicaram uma prevalência de $87,34 \%$ e $100 \%$ de $A$. (P.) longa em mugilídeos da Baía da Babitonga para M. curema e para $M$. liza, respectivamente. Devido ao risco zoonótico, é recomendado o uso de congelamento ou calor antes do consumo.

Palavras-chave: Baía da Babitonga; Mugil curema; Mugil liza; fagicolose; zoonoses.

ABSTRACT: Ascocotyle (Phagicola) longa is the etiological agent of human phagicolosis, a zoonosis. Mugilids are considered the second intermediate hosts of the digenean $A$. (P.) longa, the first one being the snail Cochliopidae Heleobia australis. In Mugil liza, from the Brazilian seaside $100 \%$ of prevalence of $A$. (P.) longa metacercariae cysts were reported. The aim of this study was to verify the prevalence rate of $A$. (P.) longa metacercariae in mugilids from Babitonga Bay, North of the Santa Catarina State. A total of 119 mugilids, $79 \mathrm{M}$. curema and $40 \mathrm{M}$. liza were fished in the Babitonga Bay from November 2015 to October 2017. Muscle tissue and liver samples were collected for parasitological analysis. The samples were grounded in a blender, the content was diluted in water, seived and settled. After discharged the supernatant, all settled material was examined in a stereoscopical microscope to search for $A$. (P.) longa metacercariae cysts. The results indicated $87.34 \%$ and $100 \%$ prevalence of $A$. (P.) longa in mugilids $M$. curema and $M$. liza from Babitonga Bay, respectively. Due to zoonotic risk, it is recommended the use of freezing or heating before consumption.

Keywords: Babitonga Bay; Mugil curema; Mugil liza; phagicolosis; zoonosis. 


\section{INTRODUÇÃO}

A maioria do pescado, entre eles os mugilideos, abrigam formas parasitárias, sendo que algumas apresentam risco zoonótico. Partes comestíveis, músculo, por exemplo e vísceras de tainha (Mugil liza Valenciennes, 1836) e de parati (Mugil curema Valenciennes, 1836) podem conter cistos de metacercárias de Ascocotyle (Phagicola) longa e a contaminação por humanos ocorre, principalmente, quando consumidos crus, mal cozidos ou em condições de higiene inadequadas. A identificação de formas imaturas e determinação da carga parasitária, em tecidos comestíveis de pescado, bem como os hospedeiros intermediários, contribui para o esclarecimento da epidemiologia da helmintofauna e avaliação do risco zoonótico (CITTI et al., 2014; FERRAZ et al., 2014).

O digenético $A$. (P.) longa, agente da fagicolose humana tem um ciclo de vida com dois hospedeiros intermediários, sendo que o primeiro hospedeiro intermediário é o caracol Cochliopidae Heleobia australis e o segundo hospedeiro intermediário os mugilídeos, tainhas e paratis. A ocorrência de $A$. (P.) longa, nas suas diversas fases de vida, está relacionada com estuários e lagoas costeiras, habitat de mugilídeos e do referido caracol. A alta prevalência, de até $100 \%$, de metacercárias de $A$. (P.) longa encistadas nestes peixes, encontradas até mesmo em áreas urbanas, como na Lagoa Rodrigues de Freitas no Rio de Janeiro, corrobora o potencial impacto na saúde pública (SIMÕES et al., 2010).

A ampla distribuição geográfica do trematódeo $A$. (P.) longa e de mugilídeos, seus hospedeiros intermediários, acompanhado do aumento do consumo de peixe cru ou mal cozido, pode aumentar o risco de infecção humana por fagicolose. Não obstante o fato desta zoonose ser até mesmo subestimada, provavelmente devido à ausência de sinais clínicos e sintomas característicos em humanos (NAMBA et al., 2012; SANTOS et al, 2013).

Chieffi et al. (1990) relataram a infecção de Phagicola sp., atualmente $A$. (P.) longa, em um paciente humano, da região de Cananéia, Estado de São Paulo, com hábito de ingerir mugilídeos em preparações cruas. Chieffi et al. (1992) relataram parasitismo por Phagicola sp. em humanos e em cão, em Registro, Estado de São Paulo, diagnosticado por exame de fezes. Almeida Dias e Woiciechovski (1994) relataram fagicolose em humanos, por exame fecal, em Cananéia e em Registro, SP, corroborando a importância em saúde pública.

O objetivo deste estudo foi verificar a prevalência de cistos contendo metacercárias de $A$. (P) longa em mugilídeos destinados ao consumo humano, pertencentes às espécies de M. liza e M. curema, provenientes da Baía da Babitonga, litoral Norte do estado de Santa Catarina, Brasil.

\section{MATERIAL E MÉTODOS}

O estudo foi realizado em 119 mugilídeos, sendo 79 espécimes de $M$. curema e $40 \mathrm{M}$. liza, adquiridos em estabelecimento comercial, no município de São Francisco do Sul, Santa Catarina, Brasil, pescados na Baía da Babitonga $\left(26^{\circ} 02^{\prime}-26^{\circ} 28^{\prime} S\right.$ e $48^{\circ} 28^{\prime}-$ $\left.48^{\circ} 50^{\prime} \mathrm{W}\right)$, no período de novembro de 2015 a outubro de 2017.

A biometria, medidas de comprimento padrão $(\mathrm{CP})$, comprimento total (CT), em centímetros ( $\mathrm{cm}$ ) e massa em kilogramas $(\mathrm{kg})$, dos exemplares, foi realizada em laboratório. A determinação do sexo dos peixes foi realizada no momento das necropsias, estas, seguiram recomendações de Namba et al. (2012), Citti et al. (2014) e 
Ferraz et al. (2014). Foram colhidas e pesadas amostras de tecido muscular $(5,22 \pm 2,12 \mathrm{~g})$ e do fígado de cada peixe.

Os fragmentos de músculo e o fígado foram triturados em liquidificador doméstico a 2.000 rpm por 20 segundos com $240 \mathrm{~mL}$ de água. O conteúdo processado foi coado em tamis $(600$ $\mu \mathrm{m})$, em um copo para decantação. Após 20 minutos o sobrenadante foi desprezado e o sedimento, em frações equivalentes a $6 \mathrm{~mL}$ por amostra, foi transferido para uma placa de Petri, e analisado em microscópio estereoscópico, para a pesquisa de cistos contendo metacercárias de A. (P.) longa.

Os cistos contendo metacercárias de A. (P.) longa (Figura 1) foram identificados conforme Scholz (1999), Scholz et al. (2001), Simões et al. (2010), Martorelli et al. (2012) e Santos et al. (2013). Os mugilídeos, $M$. liza e $M$. curema foram identificados segundo Menezes (1983), Menezes et al. (2010), Menezes et al. (2015).

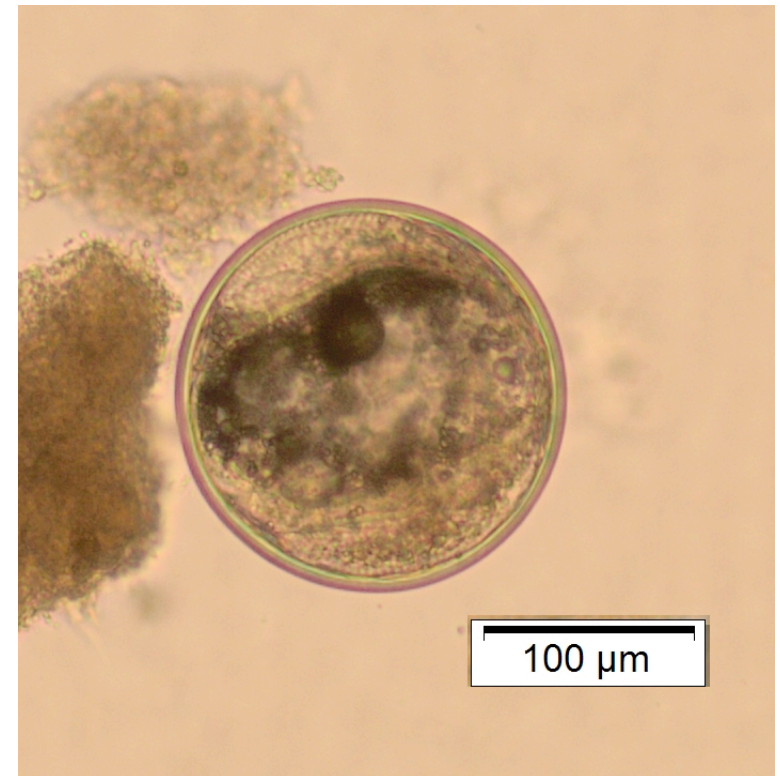

Figura 1 - Cisto contendo metacercária de Ascocotyle (Phagicola) longa.

Os descritores parasitológicos e epidemiológicos seguiram as recomendações de Bush et al. (1997). Os dados foram submetidos, inicialmente, ao teste de Shapiro-Wilk, para verificar o tipo de distribuição, sua normalização ou não. O conjunto de dados considerados normais, na sua distribuição, foram submetidos à análise de variância, caso esta acusasse diferença, era aplicado o teste de Tukey, para verificar qual ou quais conjuntos eram diferentes. Caso 0 teste de Shapiro-Wilk resultasse em dados fora do padrão de normalidade, o conjunto de dados era submetido à normalização usando a transformação tipo box-cox. Para comparação de conjuntos considerados fora da normalidade, mesmo após transformação, foram aplicados os testes de Kruskal-Wallis e Mann-Whitney. Para auxiliar nestas análises e na estatística descritiva foram utilizados os software estatísticos Past e R (HAMMER et al., 2001; PETRIE; WATSON, 2009; ANDRADE; OGLIARI, 2013; R CORE TEAM, 2017).

\section{RESULTADOS}

Os valores médios, os desvios padrões e a prevalência de metacercárias de A. (P.) longa encistadas em $M$. curema e $M$. liza, na Baía da Babitonga, litoral Norte de Santa Catarina, no período de novembro de 2015 a outubro de 2017 está apresentada nas Tabelas 1, 2 e 3. A prevalência de cistos contendo metacercárias de $A$. (P.) longa, em $M$. curema e $M$. liza, no litoral brasileiro, está expressa na Tabela 4, pois Mugil platanus e $M$. liza, são consideradas a mesma espécie, sendo aqui referida como M. liza.

Observou-se correlação entre a biometria e sexo dos espécimes e a prevalência de $A$. (P.) longa em mugilídeos. Em M. liza a correlação entre a biometria, comprimento total, comprimento padrão e a massa dos peixes e a intensidade de cistos contendo metacercárias de $A$. (P.) longa 
no fígado e amostras de tecido muscular foi fraca $(r=<0,40)$, nula $(r=0)$ ou negativa $(r=<0)$. Já, entre as fêmeas de $M$. liza houve uma forte correlação $(r>0,70)$ entre a intensidade de infecção no fígado e CT e CP, além de uma moderada correlação $(0,40<r<0,70)$ entre os peixes de sexo indeterminado. biometria, CT e CP ( $r=0,54$ e $r=0,50$ respectivamente) e a intensidade de cistos contendo metacercárias de $A$. (P.) longa no fígado, em fêmeas, foi do tipo moderada. As demais correlações foram negativas $(r<0)$, nulas $(r=0)$ e fracas $(r<0,40)$.

Em M. curema a correlação entre a

Tabela 1 - Prevalência de Ascocotyle (Phagicola) longa em mugilídeos provenientes da Baía da Babitonga, SC, de novembro de 2015 a outubro de 2017.

\begin{tabular}{|c|c|c|c|c|c|c|c|c|}
\hline & \multicolumn{4}{|c|}{ M. curema } & \multicolumn{4}{|c|}{ M. liza } \\
\hline & \multirow[t]{2}{*}{$\mathrm{n}$} & \multicolumn{3}{|c|}{ Cistos contendo $A$. (P.) longa } & \multirow[t]{2}{*}{$\mathrm{n}$} & \multicolumn{3}{|c|}{ Cistos contendo $A$. (P.) longa } \\
\hline & & Fígado & Músculo & Total & & Fígado & Músculo & Total \\
\hline Fêmeas & $\begin{array}{c}19 \\
(24,05 \%)\end{array}$ & $\begin{array}{c}11 / 19^{(1)} \\
(57,90 \%)\end{array}$ & $\begin{array}{c}16 / 19 \\
(84,21 \%)\end{array}$ & $\begin{array}{c}17 / 19 \\
(89,47 \%)\end{array}$ & $\begin{array}{c}14 \\
(35,00 \%)\end{array}$ & $\begin{array}{c}13 / 14 \\
(92,86 \%)\end{array}$ & $\begin{array}{c}10 / 14 \\
(71,43)\end{array}$ & $\begin{array}{c}14 / 14 \\
(100,00 \%)\end{array}$ \\
\hline Machos & $\begin{array}{c}14 \\
(17,72 \%)\end{array}$ & $\begin{array}{c}09 / 14 \\
(64,29 \%)\end{array}$ & $\begin{array}{c}11 / 14 \\
(78.57)\end{array}$ & $\begin{array}{c}13 / 14 \\
(92,86 \%)\end{array}$ & $\begin{array}{c}19 \\
(47,50 \%)\end{array}$ & $\begin{array}{c}18 / 19 \\
(94,74)\end{array}$ & $\begin{array}{c}11 / 19 \\
(57,89 \%)\end{array}$ & $\begin{array}{c}18 / 19 \\
(94,74 \%)\end{array}$ \\
\hline $\begin{array}{l}\text { Sexo } \\
\text { indeterminado }\end{array}$ & $\begin{array}{c}46 \\
(58,23 \%)\end{array}$ & $\begin{array}{c}26 / 46 \\
(56,52 \%)\end{array}$ & $\begin{array}{c}33 / 46 \\
(71,74 \%)\end{array}$ & $\begin{array}{c}36 / 46 \\
(78,26 \%)\end{array}$ & $\begin{array}{c}07 \\
(17,50 \%)\end{array}$ & $\begin{array}{c}05 / 07 \\
(71,43 \%)\end{array}$ & $\begin{array}{c}04 / 07 \\
(57,14 \%)\end{array}$ & $\begin{array}{c}6 / 7 \\
(85,71)\end{array}$ \\
\hline Total & $\begin{array}{c}79 \\
(100,00 \%)\end{array}$ & $\begin{array}{c}46 / 79 \\
(58,23 \%)\end{array}$ & $\begin{array}{c}60 / 79 \\
(75,95 \%)\end{array}$ & $\begin{array}{c}66 / 79 \\
(83,54 \%)\end{array}$ & $\begin{array}{c}40 \\
(100,00 \%)\end{array}$ & $\begin{array}{c}36 / 40 \\
(90,00 \%)\end{array}$ & $\begin{array}{c}25 / 40 \\
(62,50 \%)\end{array}$ & $\begin{array}{c}38 / 40 \\
(95,00)\end{array}$ \\
\hline
\end{tabular}

(1)peixes parasitados/peixes examinados.

Tabela 2 - Valores médios e desvio padrão da taxa de prevalência de cistos contendo Ascocotyle (Phagicola) longa em Mugil curema provenientes da Baía da Babitonga, SC, de novembro de 2015 a outubro de 2017.

\begin{tabular}{lccccccccc}
\hline \multicolumn{10}{c}{ Mugil curema } \\
\hline & \multicolumn{2}{c}{ Fêmeas } & \multicolumn{2}{c}{ Machos } & \multicolumn{2}{c}{ Sexo } \\
& Fígado & Músculo & Fígado & Músculo & Fígado & Músculo & Fígado & Músculo \\
Média & 39,67 & 14,50 & 09,78 & 13,27 & 08,87 & 09,33 & 15,50 & 11,43 \\
Desvio padrão & 120,48 & 15,14 & 10,47 & 15,46 & 18,86 & 10,70 & 58,28 & 12,9 \\
\hline
\end{tabular}

Peso do fígado e amostra do tecido muscular padronizado para $5 \mathrm{~g}$.

Tabela 3 - Valores médios e desvio padrão da taxa de prevalência de cistos contendo Ascocotyle (Phagicola) longa em Mugil liza, provenientes da Baía da Babitonga SC, de novembro de 2015 a outubro de 2017.

\begin{tabular}{lcccccccc}
\hline & \multicolumn{2}{c}{ Fêmea } & \multicolumn{2}{c}{ Macho } & \multicolumn{2}{c}{ Sexo } & \multicolumn{2}{c}{ Total } \\
& Fígado & Músculo & Fígado & Músculo & Fígado & Músculo & Fígado & Músculo \\
Média & 13,63 & 2,63 & 31,85 & 2,30 & 7,27 & 1,61 & 21,57 & 2,23 \\
Desvio padrão & 13,08 & 2,22 & 56,34 & 1,65 & 6,13 & 0,99 & 40,81 & 1,82 \\
\hline
\end{tabular}

Peso do fígado e amostra do tecido muscular padronizado para $5 \mathrm{~g}$. 
Tabela 4 - Prevalência de cistos contendo metacercárias Ascocotyle (Phagicola) longa em mugilídeos, no litoral brasileiro.

\begin{tabular}{lccc}
\hline \multicolumn{1}{c}{ Autores } & Local & Mugilídeo & Prevalência (\%) \\
\hline Almeida Dias e Woiciechovski (1994) & Cananéia e Registro SP & M. curema e M. liza & 95 a 100 \\
Antunes e Almeida Dias (1994) & Santos e São Vicente SP & M. curema e M. liza & 100 \\
Conceição et. al (2000) & Belém, PA & Mugil sp & 86,6 \\
Knoff e Luque (1997) & Rio de Janeiro, RJ & M. platanus & 35,3 \\
Ranzani-Paiva e Tavares-Dias (2002) & Cananéia SP & M. platanus & 99 \\
Oliveira et al. (2007) & Cananéia SP & M. platanus & 100 \\
Namba et al. (2012) & lguape SP & M. liza & 100 \\
Citti et al. (2014) & São Paulo SP & M. liza & 100 \\
Rodrigues et al., (2015) & Litoral de São Paulo & M. platanus & 100 \\
\hline
\end{tabular}

\section{DISCUSSÃO}

Com exceção de duas pesquisas, realizadas em 1994 e 1997 (Tabela 4), todas as demais, a partir de 2002, indicam prevalência de $100 \%$ de cistos contendo metacercárias de $A$. (P.) longa ou bem próximo deste número, $99 \%$. No presente estudo, a prevalência de cistos contendo metacercárias de $A$. (P.) longa, durante o período estudado, foi de $83,54 \%$ para $M$. curema e $95 \%$ para $M$. liza, sem considerar o sexo dos peixes estudados.

Em pesquisas correlatas foram encontrados cistos, contendo metacercárias do digenético $A$. (P.) longa, na musculatura do corpo, coração, estômago, fígado, vesícula biliar, rim, baço, gônadas, cérebro e mesentério, bem como em "pool de vísceras" de mugilídeos, com uma prevalência de até 100\% (SCHOLZ et al., 2001; RANZANI-PAIVA e TAVARES-DIAS, 2002; SIMÕES et al., 2010; SANTOS et al., 2013, FERRAZ et al., 2014 e RODRIGUES et al., 2015) (Tabela 4). No presente estudo, a opção foi por investigar os cistos em fragmentos da musculatura e no fígado, tecidos normalmente consumidos pela população local, na região pesquisada. O músculo é consumido na forma de filés, preparados por cocção ou até na forma crua como sushi e sashimi, sendo que o fígado é consumido após cocção.

A pesquisa de cistos, contendo $A$. $(P)$ longa, em outros tecidos, que não o muscular, como o hepático, por exemplo, também é importante, para dimensionar a distribuição dos cistos em diferentes tecidos e órgãos do peixe, uma vez que o mugilídeo é, na epidemiologia do digenético, o segundo hospedeiro intermediário e, pode ser ingerido na sua totalidade ou em frações, pelos seus predadores, em estudos futuros é importante a investigação da distribuição dos cistos, contendo metacercárias de $A$. (P.) longa em outros órgãos.

Uma prevalência de até $100 \%$ de metacercárias encistadas de $A$. (P.) longa foi encontrada na musculatura do corpo, coração, estômago, fígado, rim, baço, gônadas e mesentério de tainhas $(M$. liza), na área urbana do Rio de Janeiro e indica o potencial impacto na saúde pública, do consumo de mugilídeos, de forma inadequada (SIMÕES et al., 2010). Embora os resultados da presente pesquisa, conforme demonstrado nas Tabelas 2 e 3, para $M$. curema e para M. liza, respectivamente, não tenham chegado a $100 \%$, ficaram próximos do observado por Simões et al. (2010), sendo que, também, a Baía da Babitonga é próxima 
a áreas urbanas no litoral Norte Catarinense. Portanto, merece os mesmos cuidados com relação à saúde pública, pois como foi citado, as metacercárias têm potencial zoonótico.

Em pesquisa realizada na Lagoa Rodrigo de Freiras, no Rio de Janeiro, com M. liza, Santos et al. (2013) encontraram cistos contendo metacercárias de A. (P.) longa parasitando $100 \%$ dos peixes examinados. Porém esta prevalência variou entre órgãos, sendo $100 \%$ no baço, $98 \%$ no coração, $97 \%$ na parede do intestino e no fígado, $87 \%$ no músculo, $77 \%$ na parede do estômago, $47 \%$ no cérebro, $30 \%$ nas gônadas e $30 \%$ na vesícula biliar, sendo que não foram encontrados cisto nos olhos e nas brânquias. Também não foi observada variação sazonal na intensidade parasitária. Apesar de o presente estudo restringir a pesquisa ao fígado e ao tecido muscular, observou-se diferença $(P<0,05)$ na intensidade de infecção entre estes órgãos. Em $M$. curema a intensidade foi maior no tecido muscular e em M. liza foi maior no fígado, inclusive. O motivo desta distribuição desigual carece de investigação. No presente estudo, também não se observou variação durante o período estudado, apenas uma elevação na intensidade da infecção em fevereiro, em $M$. curema. Ressalte-se que a pesca de $M$. liza, na região estudada, é sazonal, de abril a outubro, em função da sua migração reprodutiva.

Citti et al. (2014), encontraram $100 \%(92 / 92)$ de presença de cistos contendo metacercárias de $A$. (P.) longa em amostras de musculatura e $80,43 \%$ (74/92) no "pool" de vísceras de M. liza, em São Paulo. Simões et al. (2010), Santos et al. (2013), Citti et al. (2014) e Ferraz et al. (2014), baseados em resultados prévios, desaconselham a utilização de pratos preparados a base de mugilídeos crus ou mal cozidos. Os resultados aqui apresentados corroboram com esta recomendação.

A Baía da Babitonga, litoral Norte Catarinense, proporciona condições para o desenvolvimento do ciclo de $A$. (P.) longa e portanto, também para a prevalência de cistos contendo metacercárias do digenético citado em mugilídeos. Esta baía abriga aves piscívoras e mamíferos marinhos e terrestres, com potencial predador, que podem ser hospedeiros definitivos do digenético, como relatado por Barros et al. (2002).

Os estudos de Santos et al. (2013) não mostraram correlação significativa entre o comprimento do peixe e a intensidade de infecção por $A$. (P.) longa. No presente estudo observou-se correlação entre a biometria e sexo dos espécimes e a prevalência de $A$. (P.) longa, nos mugilídeos estudados. Estas correlações foram maiores nos exemplares fêmeas em relação a machos e peixes com sexo indeterminado.

Antunes e Almeida Dias (1994), em Santos e São Vicente, SP, encontraram $100 \%$ de $M$. liza e $M$. curema contaminados por A. (P.) longa (=Phagicola longa), no fígado e coração. No tecido muscular encontraram 5,26\% das amostras contaminadas. Também observaram que as metacercárias encistadas conservaram-se vivas, mesmo em refrigeração, até três dias no peixe e até sete dias em lâminas preparadas para microscopia. $\mathrm{Na}$ presente pesquisa foi encontrado entre $75,95 \%$ de prevalência de $A$. ( $P$.) longa em M. curema e $62,50 \%$ em $M$. liza, em tecido muscular. Há uma diferença entre os resultados, porém a metodologia da pesquisa dos cistos foi diferente, bem como há a variação espaço temporal a se levar em conta, mas o que chama a tenção no trabalho, em Santos e São Vicente, é a sobrevivência das metacercárias, mesmo sob refrigeração, 
demonstrando que este tipo de conservação não inativa o parasito, necessitando de outros métodos como, por exemplo, congelamento ou tratamento pelo calor.

A descrição da prevalência de parasitos proporciona boa avaliação do risco sanitário do consumo de pescado (FERRAZ et al., 2014). Desta forma, o presente trabalho reúne dados sobre a prevalência de $A$. (P.) longa, em mugilídeos, na Baía da Babitonga, no período de dois anos, sendo portanto é um bom indicativo de risco sanitário.

\section{CONCLUSÃO}

A prevalência de metacercárias encistadas de $A$. (P.) longa em mugilídeos pescados na Baía da Babitonga, litoral Norte catarinense, no período de novembro de 2016 a outubro de 2017, em amostras de músculo e no fígado, foi de $87,34 \%$ para $M$. curema e $100 \%$ para M. liza. Portanto, estes mugilídeos, antes do consumo, devem passar por processo térmico, seja congelamento ou tratamento pelo calor, pois as metacercárias do trematódeo digenético têm potencial zoonótico.

\section{REFERÊNCIAS}

ALMEIDA

DIAS,

E.R.; WOICIECHOVSKI, E. Ocorrência da Phagicola longa (Trematoda: Heterophyidae) em mugilídeos e no homem, em Registro e Cananéia, SP. Higiene Alimentar v.8, n.31, p.43-46, 1994.

ANDRADE, D. F.; OGLIARI, P. J. Estatística para ciências agrárias $e$ biológicas: com noções de experimentação. 3. ed. rev. amp. Florianópolis: Ed. da UFSC, 2013,

$478 p$.

ANTUNES, S.A.; ALMEIDA DIAS, E.R. de Phagila longa (Trematoda:
Heterophyidae) em mugilídeos estocados resfriados e seu consumo cru em São Paulo, SP. Higiene Alimentar, v.8, n.31, p.41-42, 1994.

BUSH, A.O.; LAFFERTY, K.D.; LOTZ, J.M. et al. Parasitology meets ecology on its own terms: Margolis et al. revisited. Journal of Parasitology, v.83, p.575-583, 1997.

BARROS, L.A.; ARRUDA, V.S. de; GOMES, D.C. et al. First natural infection by Ascocotyle (Phagicola) longa Ransom (Digenea, Heterophyidae) in an avian host, Ardea cocoi Linnaeus (Aves, Ciconiiformes, Ardeidae) in Brazil. Revista Brasileira de Zoologia, v.19, n.1, p.151-155, 2002.

CHIEFFI, P.P.; LEITE, O.H.; DIAS, R.M.D.S. et al. Human parasitism by Phagicola sp. (Trematoda, Heterophyidae) in Cananéia, São Paulo State, Brazil. Revista do Instituto de Medicina Tropical de São Paulo, v.32, n.4, p.285-288, 1990.

CHIEFFI, P.P.; GORLA, M.C,; TORRES, D.M. et al. Human infection by Phagicola sp. (Trematoda, Heterophyidae) in the municipality of Registro, São Paulo State, Brazil. The American Journal of Tropical Medicine and Hygiene, v.95, n.5, p.346-348, 1992.

CITTI, A.L.; RIBEIRO, N.A.S.; TELLES, E.O. et al. Ascocotyle (Phagicola) longa parasitando tainhas (Mugil liza, Valenciennes, 1836) em São Paulo: ocorrência, importância na saúde pública e estratégias de controle. Revista de Educação Continuada em Medicina Veterinária e Zootecnia do CRMV-SP, v.12, n.3, p.36-43, 2014.

CONCEIÇÃO, J.C.S. da; SÃO CLEMENTE, S.C. da; MATOS, E. Ocorrência de Phagicola longus (Ransom, 1920) Price, 1932 em tainhas (Mugil sp.) comercializadas em Belém, 
Estado do Pará. Revista de Ciências Agrárias. n.33, p.97-101, 2000.

FERRAZ, R.R.N.; NAMBA, T.K.; NIGRO, C.A. Comparação entre os métodos de extração de metacercárias de Ascocotyle sp (Trematoda: Digenea) dos tecidos de Mugil liza Valenciennes, 1836 (Teleostei: Mugilidae). Ciência Animal Brasileira, v.15, n.3, p.354-361, 2014.

HAMMER, Ø.; HARPER, D. A. T.; RYAN, P .D. PAST: Paleontological statistics software package for education and data analysis. Palaeontologia Electronica. v. 4, n1, p.1-9, 2001.

KNOFF, M.; LUQUE, J.L. Community ecology of the metazoan parasites of grey mullets, Mugil platanus (Osteichthyes: Mugilidae) from the littoral of the State of Rio de Janeiro, Brazil. Revista Brasileira de Biologia, v.57, n.3, p.441-454, 1997.

MARTORELLI, S.R.; LINO, A.; MARCOTEGUI, P. et al. Morphological and molecular identification of the fishborne metacercaria of Ascocotyle (Phagicola) longa Ransom, 1920 in Mugil liza from Argentina. Veterinary Parasitology, v.190, n.3-4, p.599-603, 2012.

MENEZES, N.A. Guia prático para conhecimento e identificação de tainhas e paratis (Pisces, Mugilidae) do litoral brasileiro. Revista Brasileira de Zoologia, v.2, n.1,

p.1-12, 1983.

MENEZES, N.A.; OLIVEIRA, C. de; NIRCHIO, M. An old taxonomic dilemma: the identity of the western south Atlantic lebranche mullet (Teleostei: Perciformes: Mugilidae). Zootaxa, v.2519, p.59-68, 2010.

MENEZES, N.A.; NIRCHIO, M.; OLIVEIRA C. et al,. Taxonomic review of the species of Mugil (Teleostei: Perciformes: Mugilidae) from the Atlantic
South Caribbean and South America, with integration of morphological, cytogenetic and molecular data. Zootaxa, v.3918, n.1, p.001-038, 2015.

NAMBA, T.K.; MADI, R.R.; UETA, M.T. Ascocotyle sp. metacercariae (Digenea: Heterophyidae) in tissues of mullets Mugil liza and Mugil curema (Osteichthyes: Mugilidae) collected in the fish trade of the Iguape City, São Paulo, Brazil. Neotropical Helminthology, v.6, n.2, p.271-275, 2012.

OLIVEIRA, S.A.; BLAZQUEZ, F.J.H.; ANTUNES, S.A. et al. Metacercárias de Ascocotyle (Phagicola) longa Ransom, 1920 (Digenea: Heterophyidae), em Mugil platanus, no estuário de Cananéia, SP, Brasil. Ciência Rural, v.37, n.4, p.1056-1059, 2007.

PETRIE, A.; WATSON, P. Estatística em ciência animal e veterinária. São Paulo: Roca, 2009, 236p.

$R$ CORE TEAM. R: A language and environment for statistical computing. R Foundation for Statistical Computing, Vienna, Austria, 2017. https://www.R-project.org/.

RANZANI-PAIVA, M.J.T.; TAVARESDIAS, M. Eritrograma, relação viscerosomática, hepatosomática e esplenosomática em tainhas Mugil platanus Günther (Osteichthyes, Mugilidae) parasitadas. Revista Brasileira de Zoologia, v.19, n.3, p.807-818, 2002.

RODRIGUES, M.V.; PÉREZ, A.C.A. de; MACHADO, T. M. et al. Research of Ascocotyle (Phagicola) longa in Heat Treated Fillets of Mullet (Mugil platanus). Fisheries and Aquaculture Journal, v.6, n.1, p.1-3, 2015.

SANTOS, C.P.; LOPES, K.C.; COSTA, V.S. et al. Fish-borne trematodosis: Potential risk of infection by Ascocotyle (Phagicola) longa (Heterophyidae). 
Veterinary Parasitology, v.193, p.302306, 2013.

SCHOLZ, T. Taxonomic study of Ascocotyle (Phagicola) longa Ransom, 1920 (Digenea: Heterophyidae) and related taxa. Systematic Parasitology, v.43, p.147-158, 1999.

SCHOLZ, T.; AGUIRRE-MACEDO, M.L.; SALGADO-MALDONADO, G. Trematodes of the family Heterophyidae (Digenea) in Mexico: a review of species and new host and geographical records. Journal of Natural History, v.35, n.12, p.1733-1772, 2001.

SIMÕES, S.B.; BARBOSA, H.S.; SANTOS, C.P. The life cycle of Ascocotyle (Phagicola) longa (Digenea: Heterophyidae), a causative agent of fish-borne trematodosis. Acta Tropica, n.113, p.226-233, 2010. 